\title{
DER EINFLUSS VON PREISERHÖHUNGEN AUF \\ KONSUMENTENEINSTELLUNGEN: EINE EXPERIMENTELLE STUDIE
}

Dr. Heiner Evanschitzky

Lehrstuhl für Distribution und Handel

Marketing Centrum Münster (MCM)

Universität Münster

Am Stadtgraben 13-15

48143 Münster

Tel. 0251-83-22036

Fax: 0251-83-22032

evanschitzky@ifhm.de

Dipl.-Kffr. Sabine Tönnis

Lehrstuhl für Distribution und Handel

Marketing Centrum Münster (MCM)

Universität Münster

Am Stadtgraben 13-15

48143 Münster

Dr. David Woisetschläger

Lehrstuhl für Marketing

Universität Dortmund

Otto-Hahn-Str. 6

44227 Dortmund

Prof. Dr. Dieter Ahlert

Lehrstuhl für Distribution und Handel

Marketing Centrum Münster (MCM)

Universität Münster

Am Stadtgraben 13-15

48143 Münster 


\begin{abstract}
Unternehmen neigen vor allem in gesättigten Märkten zum mengenorientierten Preiswettbewerb und setzen Preissenkungen vorzugsweise zur Profilierung ein, wodurch die Preiskriege und der Verdrängungswettbewerb weiterhin zunehmen. Preis- und Wertschöpfungsspielräume werden jedoch trotz der hohen Gewinnpotenziale bislang von Unternehmen nicht hinreichend ausgenutzt, da negative Reaktionen von Seiten der Konsumenten befürchtet werden. Vor diesem Hintergrund ist es das Ziel dieses Beitrags, zu untersuchen, wie ein nominal gleicher Preisanstieg durch geeignete kommunikationspolitische Maßnahmen begleitet werden kann, um eine negative Beeinflussung der wahrgenommenen Preisfairness zu minimieren.
\end{abstract}




\section{Einleitung}

Der Preis ist einer der Erfolg versprechendsten Gewinntreiber für Händler. Eine einprozentige Preiserhöhung steigert den Betriebsgewinn durchschnittlich um 11,1\%, während eine einprozentige Senkung der variablen Kosten lediglich zu einer durchschnittlichen Gewinnsteigerung von 7,8\% führt (Huckemann/Dinges 1998, S. 22). Dennoch sind Preiskriege und Verdrängungswettbewerbe nach wie vor an der Tagesordnung im deutschen Handel. Dies wird noch dadurch verstärkt, dass Unternehmen versuchen, sich über Preissenkungen zu profilieren. Doch letztendlich sind dadurch der Unternehmenserfolg und insbesondere die Marke gefährdet. Zudem sind Gewinnsteigerungspotenziale durch Kostensenkungen und Rationalisierungen mittlerweile weitgehend ausgeschöpft (Koschate 2002, S. 1; Simon/von der Gathen/Daus 2006, S. 272). Daher erscheint es eine überlegenswerte Alternative zu sein, über Preiserhöhungsstrategien nachzudenken. Insbesondere vor dem Hintergrund der schlechten Preiskenntnis der Konsumenten, die Preise überwiegend überschätzen (vgl. für eine Übersicht $\mathrm{zu}$ Preiswissensstudien Evanschitzky/Kenning/Vogel 2004, S. 392 ff.), erscheinen Preiserhöhungen ein adäquates Mittel zu sein, die Gewinne für Unternehmen zu steigern.

Unternehmen befürchten jedoch negative Kundenreaktionen auf Preiserhöhungen. Eine Preiserhöhung bewirkt eine direkte Verringerung der Kaufkraft des Kunden; letztlich kauft dieser ein Produkt nur dann, wenn sein subjektiv wahrgenommener Produktnutzen größer als sein Verlust (Preis) ausfällt (Simon 1992, S. 3). Aktuelle Beispiele zeigen, dass Preiserhöhungen teilweise jedoch unvermeidbar sind, falls Gewinneinbußen vermieden werden sollen. So entstanden z.B. durch die Einführung der Lkw-Maut im Jahr 2005 erhebliche Mehrkosten für die Unternehmen, die teilweise über Preiserhöhungen an die Verbraucher weitergegeben werden mussten (o. V. 2005). Auch die Anfang 2007 in Kraft getretene Mehrwertsteuererhöhung führte in einigen Branchen schon im Vorfeld zu Preiserhöhungen (o. V. 2006). Leider muss festgestellt werden, dass Preiserhöhungen in der Praxis häufig schlecht vorbereitet und hektisch umgesetzt werden. Daher enden viele mit einem Misserfolg oder werden gar ganz zurückgenommen.

Vor diesem Hintergrund ist es das Ziel dieses Beitrags, zu untersuchen, wie ein nominal gleicher Preisanstieg durch geeignete kommunikationspolitische Maßnahmen begleitet werden kann, um eine negative Beeinflussung der wahrgenommenen Preisfairness zu minimieren. Basierend auf der Equity-Theorie werden diesbezügliche Hypothesen hergeleitet und mittels eines 2x2 Szenario-basierten Konsumentenexperiments unter 84 Probanden überprüft. 


\section{Forschungsstand und Hypothesen}

\subsection{Aktueller Forschungsstand}

Aktuell existieren in der Forschung nur wenige Arbeiten, in denen Konsumentenreaktionen auf Preiserhöhungen untersucht werden, da vorwiegend auf den Einfluss von Preissenkungen fokussiert wird. Die bestehenden Arbeiten untersuchen zum einen primär den Einfluss von Preiserhöhungen auf Einstellungen der Konsumenten, wie z. B. auf die empfundene Preisfairness (vgl. für einen Überblick über die Einflussfaktoren und Auswirkungen von Preisfairness Xia/Monroe/Cox 2004). Diese Studien bauen zumeist auf den Erkenntnissen von Kahneman/Knetsch/Thaler (1986) auf, die das Konstrukt der Preisfairness nicht nur theoretisch bearbeitet, sondern auch empirisch untersucht haben. Maxwell (1995) konnte zeigen, dass die wahrgenommene Fairness der Preiserhöhung mit der Höhe des Preisanstiegs abnimmt. Zentrale Einflussfaktoren, die die Wahrnehmung der Preisfairness bei einer Preiserhöhung beeinflussen, sind u.a. die Veränderung des Gewinns des Unternehmens (Campbell 1999; Maxwell 1995), das vermutete Motiv für die Preiserhöhung (Campbell 1999), Preise der Vergangenheit, Preise von Wettbewerbern, Kosten des Produkts (Bolton/Warlop/Alba 2003) sowie das Verhalten der Wettbewerber und die Reputation des Anbieters (Campbell 1999).

Es lässt sich festhalten, dass sich die Literatur im Wesentlichen mit den negativen Effekten von Preiserhöhungen auf Konsumenten befasst. Aufgrund der positiven Erlöspotenziale, die sich aus einer Preiserhöhung ergeben, stellt sich jedoch die Frage, ob es nicht Preiserhöhungsstrategien gibt, die diese negativen Konsumentenreaktionen minimieren. Brambach (2005) gibt auf Basis von Experteninterviews und einer Paneluntersuchung Empfehlungen zur erfolgreichen Planung und Durchführung von Preiserhöhungen. Ein wesentlicher Aspekt zur Abschwächung negativer Kundenreaktionen scheint demnach in der offenen Kommunikation der Preiserhöhung zu bestehen. Eine empirische Untersuchung solcher Preiserhöhungsstrategien bzgl. deren Auswirkungen auf Konsumenten fehlt jedoch bisher. Dies soll hier mittels eines Experiments erfolgen, mit Hilfe dessen die nachfolgenden Hypothesen überprüft werden.

\subsection{Hypothesen}

Im Folgenden werden zwei Hypothesen zu den Auswirkungen einer Preiserhöhung auf die Preisfairness sowie zu den Einflussfaktoren - insbesondere die Kommunikation - aufgestellt. Zur theoretischen Fundierung der Hypothesen berufen wir uns im Wesentlichen auf die

Equity-Theorie (vgl. grundlegend Adams 1965; Austin/Walster 1975), die Einstellungsänderungen nach Preiserhöhungen erklärt. Gemäß der Equity-Theorie, die sich 
mit Fairness in sozialen Austauschbeziehungen beschäftigt (Adams 1965), wird der wahrgenommene Outcome der Austauschbeziehung mit dem aufgebrachten Input ins Verhältnis gesetzt und mit dem Outcome-Input-Verhältnis vor der Preiserhöhung verglichen. Austauschbeziehungen werden dann als fair wahrgenommen, wenn das Outcome-InputVerhältnis einer Referenzgröße, dem eigenen Outcome-Input-Verhältnis, entspricht (Homburg/Koschate 2004, S. 317 f.). Da sich durch den Preisanstieg der Outcome des Anbieters erhöht, nimmt die wahrgenommene Preisfairness ab und zwar umso stärker, je höher der Preisanstieg, also je größer der Unterschied des Vergleichs ausfällt. Die wahrgenommene Inequity führt dazu, dass eine Person innere negative Spannungen empfindet, die sie motiviert, Maßnahmen zu ergreifen, um Equity wiederherzustellen (Adams 1963; 1965). Equity kann wiederhergestellt werden, indem die Einstellung gegenüber dem Anbieter - hier die empfundene Preisfairness - so geändert wird, dass sie ebenfalls negativ wird.

$H_{1}$ : Je höher der Preisanstieg, desto geringer ist die wahrgenommene Preisfairness.

Da angenommen wird, dass Preiserhöhungen grundsätzlich negativ auf die Preisfairness wirken, soll im Folgenden der Einfluss einer wesentlichen Marketingmaßnahme, die in Kombination mit einer Preiserhöhung durchgeführt werden kann, untersucht werden: die Kommunikation. Genauer soll der Einfluss verschiedener Kommunikationsarten auf die abhängige Variable untersucht werden, da Anbieter bei Preiserhöhungen z.B. durch qualitätsimageorientierte Werbung versuchen, auf die herausragenden Komponenten des Produkts hinzuweisen. In einem ersten Pretest mit 99 Probanden wurde ein signifikanter Unterschied zwischen einer direkten, durch Werbung kommunizierten, angekündigten (Mittelwert (MW) = 4,66) sowie einer indirekten, durch PR kommunizierten Preiserhöhung ( $\mathrm{MW}=2,25)$ ermittelt [auf einer Skala von $1=$,sehr fair“ bis $7=$,sehr unfair“ (t-Wert $=$ 23,394, p < 0,01)]. Es kann vermutet werden, dass eine durch PR kommunizierte Preiserhöhung als glaubhafter wahrgenommen wird, Informationsasymmetrien abbaut und somit die Unsicherheit der Konsumenten reduziert, was zu erhöhter wahrgenommener Fairness führt (Kaas 1990). Folglich ist zu vermuten, dass negative Mundpropaganda umso geringer ausfällt, je glaubwürdiger die Preiserhöhung kommuniziert wird.

$H_{2 a}$ : Glaubwürdige Kommunikation hat einen positiven Einfluss auf die wahrgenommene Preisfairness. 
$H_{2 b}$ : Je glaubwürdiger die Kommunikation der Preiserhöhung, desto geringer ist die Bereitschaft zur negativen Mundpropaganda.

\section{Experimentelle Untersuchung}

\section{1. Darstellung der experimentellen Studie}

Zur empirischen Überprüfung der Hypothesen wurde ein Experiment in Form der SzenarioTechnik durchgeführt. Den Rahmen der Untersuchung bildet die Kategorie Süßwaren (Schokoladenriegel) im Lebensmittelhandel. Dieser Rahmen wurde bewusst gewählt, da davon ausgegangen werden kann, dass alle Probanden hinreichend Erfahrung mit den im Experiment beschriebenen Szenarien haben und daher valide Aussagen dazu treffen können. Das Experiment basiert auf einem 2x2 Between-Subjects-Design mit der „Kommunikationsart“ (klassische Werbung versus Public Relations) und der „Höhe des Preisanstiegs“ (zwei verschieden hohe Preisanstiege) als experimentelle Faktoren sowie der Preisfairness und die Bereitschaft zur negativen Mundpropaganda (bezogen auf das Produkt sowie den Händler) als zentrale abhängige Variablen. Es wurden im Juni 200684 deutschen Studenten (55,4\% weiblich) im Alter von 19 bis 30 Jahren (MW = 23,1 Jahre) als Probanden ausgewählt. Da Studenten eine relativ homogene Stichprobe darstellen, tritt weniger Störvarianz auf und somit ist eine strenge Überprüfung der Hypothesen möglich (Homburg/Koschate 2004, S. 326). Zudem weisen die Studentendaten ebenso ein akzeptables Maß an externer Validität auf (Lynch 1999) und ihr Einsatz bei Experimenten ist in der Forschung, insbesondere bei Fragestellungen zum Pricing, üblich (Maxwell 2002). Da das Experiment aus 4 Szenarien besteht, können je Szenario 21 Probanden untersucht werden (Tab.1).

\section{Tabelle 1}

\subsection{Operationalisierung der Variablen}

Im Rahmen des Experiments wurden die zwei Merkmale „Kommunikationsart“ und „Höhe des Preisanstiegs“ als unabhängige Variablen manipuliert. Zur Manipulation der Kommunikationsart wurden basierend auf dem oben geschilderten Pretest zwei verschiedene Kommunikationsmaßnahmen unterschieden. Es wurde zwischen klassischer Werbung, die die besondere Qualität des Produkts herausstellt und die Preiserhöhung ankündigt, und Public Relations unterschieden. Mit Hilfe von Public Relations wird die Preiserhöhung den 
Konsumenten transparent gemacht, um somit die Glaubwürdigkeit und das Vertrauen zu verbessern.

Für die Höhe des Preisanstiegs, dem zweiten experimentellen Faktor, gibt es in der Literatur keine genauen Vorgaben, wie dieser zu manipulieren sei. In einer empirischen Studie ermittelten Zelleken/Dellbrügge (2000, S. 18 f.), dass Preiserhöhungen bis zu 5,6\% oder solche, die unter einer Preisschwelle bleiben, bei Lebensmitteln keine negative Reaktion auf die Absatzmenge auslösen. Sollte eine Preisschwelle überschritten werden, ist es vorteilhaft, direkt unter die nächst höhere zu gehen (Graumann 1990, S. 111 f.). Da die Ermittlung der relativen Preisschwellen jedoch problematisch ist, wurde in Anlehnung an Homburg/Koschate (2003) in einem weiteren Pretest mit 99 Probanden der kleinste Preisanstieg vom aktuellen Preis der jeweiligen Produkte ermittelt, der das Kaufverhalten der Probanden beeinflussen würde. Die Preisstufen wurden mit 1,75€ (Basispreis), 1,89€ (erste Preiserhöhungsstufe) und 1,99€ (zweite Preiserhöhungsstufe) so gesetzt, dass ein Drittel der Probanden nach der ersten Stufe und zwei Drittel der Probanden nach der zweiten Stufe reagieren würden. Ebenso wurden die Preise so gesetzt, dass die Preisoptik (Diller 2000, S. 136 ff.; Monroe 2003, S. 113 ff.) eingehalten wird.

Die abhängige Variable „Preisfairness“ wird in Anlehnung an Grewal/Hardesty/Iyer (2004) mittels dreier Items gemessen, welche auf einer siebenstufigen Likert-Skala mit den Endpunkten 1 = „trifft gar nicht zu“ und 7 = „trifft voll zu“ (bzw. „überhaupt nicht fair“ und „sehr fair“) von den Probanden bewertet werden. Die Absicht zur negativen Mundpropaganda bezogen auf das Produkt bzw. den Händler wurde mit einer Skala von Singh $(1988,1990)$ mit jeweils drei Items operationalisiert.

\subsection{Ergebnisse}

Zur Überprüfung der Hypothesen wird eine mulitvariate Varianzanalyse (MANOVA) durchgeführt. Die sich anschließenden Follow-Up-ANOVAs geben Auskunft darüber, welche unabhängigen Variablen Unterschiede bei den dargestellten abhängigen Variablen hervorrufen. Mittels eines F-Tests wird geprüft, ob die Unterschiede zufällig auftreten oder ob sie signifikant sind (Janssen/Laatz 2003, S. 325). Um die Effektrichtung, durch die sich die Gruppen voneinander unterscheiden, festzustellen, werden mit Hilfe von Post-Hoc-Tests paarweise Vergleiche zwischen den Gruppen vorgenommen (zum Vorgehen vgl. Eschweiler 2006; Eschweiler/Evanschitzky/Woisetschläger 2007, S. 16).

Zunächst lässt sich feststellen, dass das Ausmaß der Preiserhöhung für sich alleine genommen keine signifikanten Unterschiede in der wahrgenommenen Preisfairness bewirkt. Die durchgeführte ANOVA ergab, dass die erste Stufe der Preiserhöhung (MW = 3,47) von den 
Probanden nicht signifikant fairer eingestuft wurde als die zweite Preiserhöhung ( $M W=3,39$ ) $(F=0,079 ; p=0,78)$. Daher muss die Hypothese $H_{1}$ abgelehnt werden .

Die Manipulation der Kommunikation ergibt, dass signifikante Mittelwertunterschiede bzgl. der abhängigen Variablen „Preisfairness“ und „Bereitschaft zu negativer Mundpropaganda gegenüber dem Händler“ vorliegen. Ferner lässt sich feststellen, dass „Public Relations“ (K2) fairer eingeschätzt wird als Werbung (K1) (Tab. 2). $\mathrm{H}_{2 \mathrm{a}}$ kann daher angenommen werden. Die Bereitschaft zur negativen Mundpropaganda ist im Fall der Kommunikation durch Werbung sowohl für das Produkt als auch für den Händler höher, jedoch ist nur der Unterschied bezogen auf den Händler signifikant. $\mathrm{H}_{2 b}$ kann daher nur teilweise angenommen werden.

\section{Tabelle 2}

Die Durchführung der multivariaten Varianzanalyse (MANOVA) sowie die daran anschließenden Follow-Up-ANOVAs zeigen, dass signifikante Interaktionseffekte zwischen „Arten der Kommunikation“ und „Preiserhöhung“ für die Bereitschaften zur negativen Mundpropaganda vorliegen ( $F=$ 4,442 für Produkt bzw. 6,419 für den Händler). Im Fall der ersten Preiserhöhungsstufe ist die Bereitschaft zur negativen Mundpropaganda gegenüber dem Produkt $(\mathrm{F}=5,787, \mathrm{p}<0,05)$ bzw. Händler $(\mathrm{F}=11.324, \mathrm{p}<0,01)$ deutlich höher, wenn die Kommunikation durch Werbung erfolgt. Für die zweite Preiserhöhungsstufe sind die Unterschiede zwischen Werbung und Kommunikation für beide abhängigen Konstrukte nicht mehr signifikant ( $F=0,314$ bzw. $F=0,128$ ). Werbung wird zwischen beiden Stufen der Preiserhöhung für beide Konstrukte signifikant unterschiedlich wahrgenommen $(F=4.188$, $\mathrm{p}<0,05$ für Produkt, $\mathrm{F}=5,070, \mathrm{p}<0,05$ für Händler), während $\mathrm{PR}$ für beide Stufen nicht signifikant unterschiedlich empfunden wird $(F=0,797$ bzw. $F=1,474)$. Die Interaktionseffekte sind in Abbildung 1 veranschaulicht.

\section{Abbildung 1}

\section{Implikationen}

Preiserhöhungen stellen ein probates Mittel zur Erlössteigerung beim Handel dar. Es besteht jedoch die Gefahr, durch eben solche Preiserhöhungen Kunden zu verlieren. Vor jeder Durchführung einer Preiserhöhung sollte als erstes die Preiselastizität der Konsumenten sowie deren Preisschwelle für das entsprechende Produkt ermittelt werden. Eine allgemeine Empfehlung, in welchem Maße ein Preis angehoben werden kann, ist nicht möglich, da dies 
kontextspezifisch (Produktart, Preiswissen der Konsumenten, Preisimage des Händlers, etc.) ist. Sollte eine Preisschwelle überschritten werden, ist es vorteilhaft, direkt unter die nächst höhere zu gehen. Eine direkte Befragung der Kunden ermöglicht eine schnelle und unkomplizierte Ermittlung von Preisschwellen.

Die hier gefundenen Ergebnisse legen nahe, dass die Durchführung einer Preiserhöhung in Verbindung mit geeigneten kommunikationspolitischen Maßnahmen des Marketing-Mix die Gefahr, negative Reaktionen der Kundenseite hervorzurufen, reduziert. Die Nutzeneinbuße durch die Preiserhöhung wird unter gewissen Voraussetzungen durch die Kommunikation einer Erklärung aufgewogen. Insbesondere scheinen Maßnahmen im Rahmen der Public Relations als sehr glaubwürdig eingeschätzt zu werden. Mit solchen lassen sich insbesondere kleinere Preiserhöhungen gut kommunizieren. $\mathrm{Zu}$ denken ist dabei insbesondere an die Chance, im Rahmen der von Unternehmen nicht selbst zu verantwortenden Kostensteigerungen, z.B. durch Umweltauflagen, Steuererhöhungen oder Qualitätssteigerungen durch bessere Rohstoffe, Preise zu erhöhen. Höchste Vorsicht ist indes geboten, wenn kleinere Preiserhöhungen mittel klassischer Werbung kommuniziert werden. Offenbar fühlen sich die Konsumenten schnell vom Unternehmen hintergangen. Vermutlich werden bei einer werblichen Kommunikation hinter kleineren Preiserhöhungen eigennützige Motive des Unternehmens vermutet, wohingegen bei der Kommunikation größerer Preiserhöhungen durch Werbung implizit „werthaltige“ Gründe mit der Maßnahme verbunden werden.

Neben der Kommunikation von Preiserhöhungen ist es natürlich auch möglich, andere Marketing-Maßnahmen einer Preiserhöhung zur Seite zu stellen. $\mathrm{Zu}$ denken ist hier insbesondere an eine Produktinnovation, die von einer Preiserhöhung begleitet wird. Dies, sowie die Übertragung der empirischen Untersuchung auf andere Kontextsituationen (andere Produktkategorie, andere Konsumentengruppe) würden helfen, die Generalisierbarkeit der Ergebnisse zu erhöhen. In der Tat besteht noch erheblicher Forschungsbedarf, um letztlich eine für Unternehmen vernünftige und von Konsumenten als fair wahrgenommene Preiserhöhungsstrategie zu entwerfen. 


\section{LITERATURVERZEICHNIS}

Adams, J.S. (1963): Toward an Understanding in Inequity, in: Journal of Abnormal and Social Psychology, Vol. 67, No. 5, pp. 422-436.

Adams, J.S. (1965): Inequity in Social Exchange, in: Advances in Experimental Social Psychology, Vol. 2, pp. 267-299.

Austin, W./Walster, E. (1975): Equity with the World: The Trans-Relational Effects of Equity and Inequity, in: Sociometry, Vol. 38, No. 4, pp. 474-496.

Bolton, L.E./Warlop, L./Alba, J.W. (2003): Consumer Perceptions of Price (Un)Fairness, in: Journal of Consumer Research, Vol. 29, No. 4, pp. 474-491.

Campbell, M.C. (1999): Perceptions of Price Unfairness: Antecedents and Consequences, in: Journal of Marketing Research, Vol. 36, No. 2, pp. 187-199.

Diller, H. (2000): Preispolitik, 3. Aufl., Stuttgart.

Eschweiler, M. (2006): Externe Referenzpreise - Eine empirisch gestützte verhaltenswissenschaftliche Analyse, Wiesbaden.

Eschweiler, M./Evanschitzky, H./Woisetschläger, M. (2007): Laborexperimente in der Marketingwissenschaft: Bestansaufnahme und Leitfaden bei varianzanalytischen Auswertungen, MCM/IAS-Arbeitspapier Nr. 46, Münster [Download erhältlich online: http://www.ias.marketing-centrum.de/literatur/index_arbeitspapiere.html].

Evanschitzky, H./Kenning, P./Vogel, V. (2004): Consumer Price Knowledge in the German Retail Market, in: Journal of Product \& Brand Management, Vol. 13, No. 6, pp. 390405.

Graumann, J. (1990): 55 Methoden und Fallbeispiel zur Durchsetzung höherer Verkaufspreise und niedriger Rabatte, 2. Aufl., München.

Grewal, D./Hardesty, D.M./Iyer, G.R. (2004): The Effects of Buyer Identification and Purchase Timing on Consumers' Perceptions of Trust, Price Fairness, and Repurchase Intentions, in: Journal of Interactive Marketing, Vol. 18, No. 4, pp. 87-100.

Homburg, C./Koschate, N. (2004): Wie reagieren Kunden auf Preiserhöhungen? Eine Untersuchung zur Rolle von wahrgenommener Fairness des Preisanstiegs und Kundenzufriedenheit, in: Marketing - Zeitschrift für Forschung und Praxis, 26. Jg., Nr. 4, S. 316-329.

Huckemann, M./Dinges, A. (1988): Euro-Preis Marketing - Wie Sie mit der richtigen Preisstrategie gewinnen, Neuwied.

Janssen, J./ Laatz, W. (2003): Statistische Datenanalyse mit SPSS für Windows, 4. Aufl., Berlin, Heidelberg, New York. 
Kahneman, D./Knetsch, J.L./Thaler, R.H. (1986): Fairness as a Constraint on Profit Seeking: Entitlements in the Market, in: The American Economic Review, Vol. 76, No. 4, pp. 728-741.

Koschate, N. (2002): Kundenzufriedenheit und Preisverhalten: Theoretische und empirisch experimentelle Analysen, Wiesbaden.

Lynch, J. (1999): Theory and External Validity, in: Journal of the Academy of Marketing Science, Vol. 27, No. 3, pp. 367-376.

Maxwell, S. (1995): What Makes a Price Increase Seem “Fair”?, in: Pricing Strategy \& Practice, Vol. 3, S. 21-27.

Maxwell, S. (2002): Rule-Based Price Fairness and its Effect on Willingness to Purchase, in: Journal of Economic Psychology, Vol. 23, No. 2, pp. 191-212.

Monroe, K.B. (2003): Pricing: Making Profitable Decisions, $3^{\text {rd }}$ ed., New York.

o. V. (2005): Getränkewirtschaft denkt über Preiserhöhungen nach, Pressemitteilung vom 08.01.2005, http://www.stern.de/wirtschaft/unternehmen.

o. V. (2006): Preiswelle rollt auf breiter Front an, Pressemitteilung vom 06.04.2006, http://www.lz-net.de/archiv.

Simon, H. (1992): Preismanagement: Analyse - Strategie - Umsetzung, 2. Aufl., Wiesbaden.

Simon, H./von der Gathen, A./Daus, P.W. (2006): Retail Pricing - Higher Profits Through Improved Pricing Processes, in: Krafft, M./Mantrala, M.K. (Eds.): Retailing in the $21^{\text {st }}$ Century - Current and Future Trends, Heidelberg, pp. 271-288.

Xia, L./Monroe, K.B./Cox, J.L. (2004): The Price is Unfair! A Conceptual Framework of Price Fairness Perceptions, in: Journal of Marketing, Vol. 68, No. 10, pp. 1-15.

Zelleken, H.-J./Dellbrügge, G. (2000): Zur Preispolitik des Lebensmittelhandels, in: Dynamik im Handel, Nr. 12, S. 10-19. 


\section{VERZEICHNISSE}

\section{Tabelle 1:}

Experimentelles Design

\begin{tabular}{|c|c|c|c|}
\hline & \multicolumn{2}{|c|}{ Experimenteller Faktor „Kommunikationsart“ } \\
\hline & & Klassische Werbung & Public Relations \\
\hline \multirow{2}{*}{ 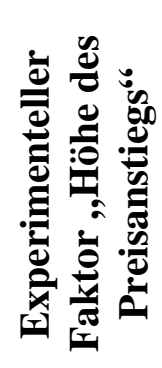 } & - & $\begin{array}{c}\text { Szenario 1: } \\
\text { Klassische Werbung x kleine } \\
\text { Preiserhöhung }\end{array}$ & $\begin{array}{c}\text { Szenario 2: } \\
\text { Public Relations x kleine } \\
\text { Preiserhöhung }\end{array}$ \\
\hline & N & $\begin{array}{c}\text { Szenario 3: } \\
\text { Klassische Werbung x große } \\
\text { Preiserhöhung }\end{array}$ & $\begin{array}{c}\text { Szenario 4: } \\
\text { Public Relations x große } \\
\text { Preiserhöhung }\end{array}$ \\
\hline
\end{tabular}




\section{Tabelle 2:}

Mittelwerte der Kommunikationsarten

\begin{tabular}{lccc}
\hline Szenario & $\begin{array}{c}\text { Mittelwert } \\
\text {,Negative } \\
\text { Mittelwert }\end{array}$ & $\begin{array}{c}\text { Mittelwert } \\
\text { „Negative } \\
\text { Mundpropaganda } \\
\text { Händler“ }\end{array}$ & $\begin{array}{c}\text { Mundpropaganda } \\
\text { Produkt“ }\end{array}$ \\
Werbung (K1) & $4,17^{*}$ & 3,07 & 3,05 \\
Public Relations (K2) & 2,97 & 2,63 & 2,42 \\
\hline
\end{tabular}

*) größere Werte stellen eine schlechter Bewertung des Konstrukts dar. 


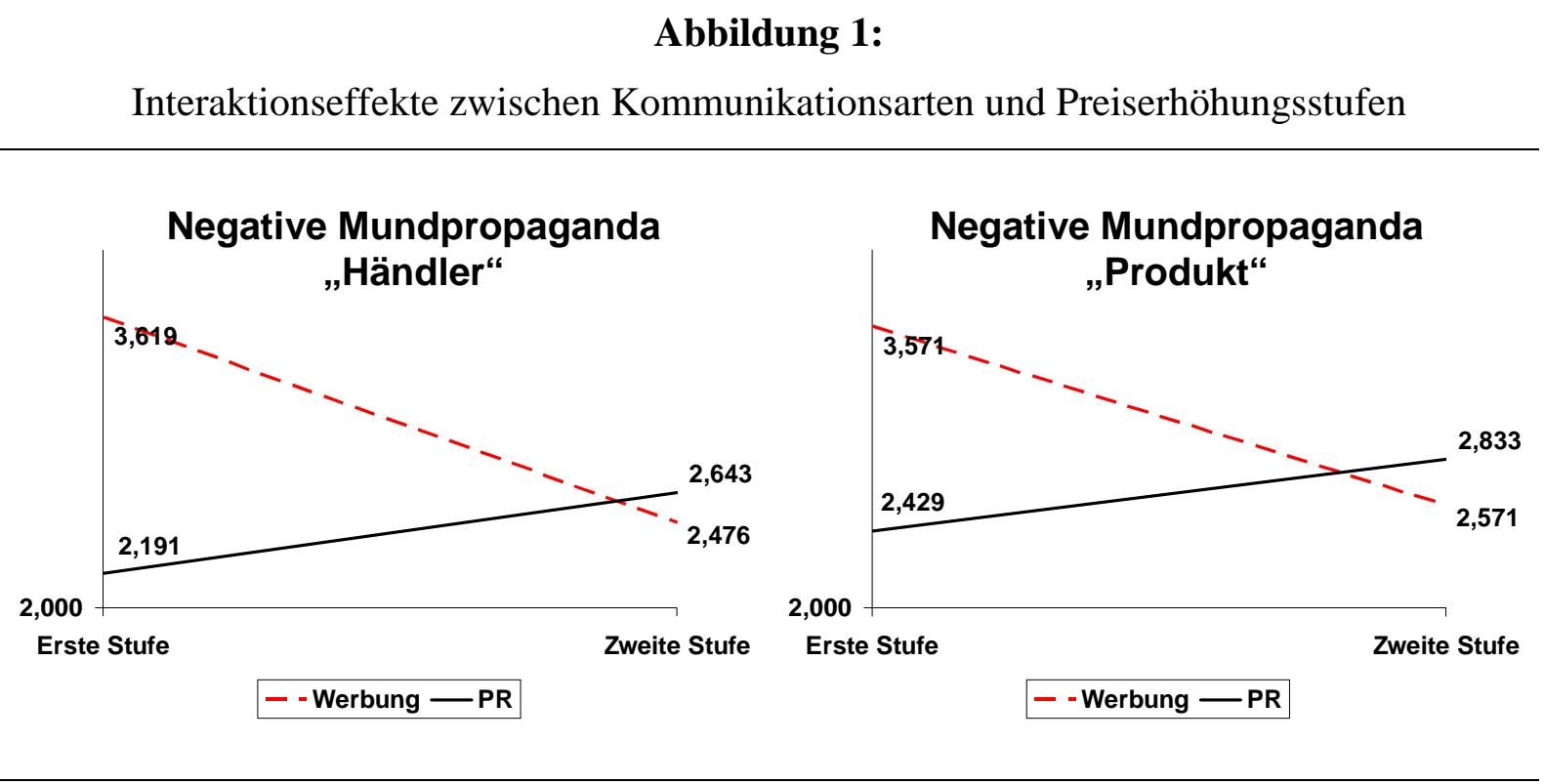

